\title{
Parametric Optimization of a non-Foster Circuit Embedded in an Electrically Small Antenna for Wideband and Efficient Performance
}

\author{
Hussein Jaafar, Dominique Lemur, Sylvain Collardey, and Ala Sharaiha, Senior Member, IEEE
}

\begin{abstract}
In this paper, we present the design of a small, wideband antenna with an embedded non-Foster circuit. The design procedure is based on modeling the antenna in the presence of the EM model of the Negative Impedance Converter (NIC) and trying to optimize the components and location of this active circuit inside the antenna. The EM modeling of the circuit provides the possibility to identify the key parameters that mostly affect the performance of the antenna in terms of bandwidth and efficiency. These parameters are then optimized accordingly. This procedure is applied on a monopole antenna which has its natural resonance at $2.2 \mathrm{GHz}$. With an embedded non-Foster circuit, the antenna is miniaturized to cover wideband frequencies around $900 \mathrm{MHz}$. Two examples are considered for different NIC topologies, after optimizing the components of the NIC, the best performance was given by the the second antenna which exhibits an impedance bandwidth of $50 \%([0.84-1.45 G \mathrm{~Hz}])$ and total efficiency of $25 \%$. The measurement results of both antennas are in good agreement with simulations showing a significant enhancement with respect to the passive antenna.
\end{abstract}

Index Terms-Electrically small atenna, non-foster matching, miniaturization.

\section{INTRODUCTION}

$\mathbf{N}$ Egative Impedance Converters (NICs) were first introduced by Linvill in 1954 [1]. The unusual reactance behaviour of such circuits, have made them an attractive alternative to solve the issues in various RF problems [2]. One of the most common application of NICs is found in matching of Electrically Small Antennas (ESAs). As the electrical size of the antenna decreases, its behaviour approaches that of a lumped element where its reactance increases significanltly and its resistance attains very low values resulting in a high $\mathrm{Q}$ factor [3], [4]. In such a case, it becomes impossible to match the antenna in a wideband using the conventional passive matching networks which are limited by the gainbandwidth theory of Bode and Fano [5], [6]. To surpass this limit, active matching networks are used. They are realized by terminating a two-port NIC with a specific impedance whose negative value is seen at the input of the NIC. This type of

Manuscript recieved 13 April, 2017. This work is supported by the European Union through the European Regional Development Fund (ERDF), and by Ministry of Higher Education and Research, Brittany and Rennes Métropole, through the CPER Project SOPHIE / STIC \& Ondes, and by the Direction Général de l'Armement (DGA). The authors are with IETR UMR CNRS 6164, Université de Rennes 1,35042 Rennes Cedex, France (e-mail: ala.sharaiha@univ-rennes1.fr)

Color versions of one or more of the figures in this letter are available online at http://ieeexplore.ieee.org.

Digital Object Identifier 10.1109/TAP.20XX.XXXXXXX
NICs is known as non-Foster circuits. The reactive part of the non-Foster circuits exhibits a negative slope as a function of frequency contrary to traditional lumped reactive elements. This special behaviour of non-Foster loads, provides higher flexibility in matching the ESAs in which it becomes easier to compensate their high reactance and hence reducing their $\mathrm{Q}$ factor [7].

In addition to ESA matching, non-Foster circuits have found application in other RF domains. The authors in [8] studied the analogy between the Negative Group Delay (NGD) and non-Foster circuits showing that it is possible generate nonFoster impedances using NGD circuit. The NGD based nonFoster elements provide superluminal characteristics which can find attractive applications in broadband metamaterials [9] and eliminitaing the beam squint in parasitic arrays [10]. Furthermore, non-Foster ciruits have also found application in enhancing the directivity bandwidth of superdirective arrays [11], [12].

Various non-Foster impedance matching of ESAs have been discussed in literature [13]-[18]. In these studies, non-Foster loads were connected at the input of the antenna. Despite the wideband achieved, it has been shown that input non-Foster matching is very sensitive [19]. A slight change in the value of the negative load can dramatically change the matching performance of the whole system. This is due to the sensitivity and instability of the NICs [20], [21]. Moreover, when targeting higher frequencies, the NIC dissipates more resistive losses which cause degradation in the overall efficiency [15]. This resistive effect will have a high impact on the efficiency since the circuit is placed directly at the feeding point. In addition, a non-Foster impedance placed at the input point can compensate the reactance of the antenna. However this does not insure a wideband matching due to the squared frequency dependence of the resistance which might impose the need for an additional input matching network [22].

Embedded non-Foster matching was adopted as an alternative for wideband ESA matching [23]-[28]. Placing the NIC inside the antenna can reduce the sensitivity of impedance matching with respect to slight changes in the non-Foster load value. Additionaly, an internal non-Foster load can provide better control on both the resistance and reactance of the antenna making it a more suitable approach for wideband matching [29]

Most of the practical non-Foster matching circuits for ESA discussed in literature are limited to taregting frequency bands below $500 \mathrm{M} \mathrm{Hz}$ [25], [30], [31] . For higher frequency bands 
(above $800 \mathrm{MHz}$ ) the ideal non-Foster value is considered in simulation, in which high simulated efficiency and impedance matching is shown [32]. The lack of practical study of nonFoster matching at higher frequency bands, is due to the fact that the design of such circuits becomes more constrained by stability, the non-linearity of the active components and the effect of their parasitic elements, which might eventually limit the possibilty to optimize the performance of the circuit. In [15], the authors have succeeded to realize a NIC producing a low negative capacitance value $(C=-0.87 p F)$ which was however associated with high resistive losses. This circuit was connected at the input of an ILA antenna to match it in a wide frequency band (above $800 \mathrm{MHz}$ ) while it suffered from a low efficiency in the lower part of the band (12\%).

In this paper we present a systematic design of a wideband electrically small monopole antenna embedded with a nonFoster circuit. The monopole is naturally resonant and matched at a $2.2 \mathrm{GHz}$. The non-Foster circuit is then embedded to miniaturize the antenna and match it in a lower frequency wideband. To accurately model the effect of the non-Foster circuit inside the antenna, the EM model of the ciruit is taken into account. By introducing the EM model of the NF circuit in the full wave simulation of the antenna, accurate results can be predicted compared to measurements. This accuracy, provides the possibility to optimize the location and the critical parameters of the NF circuit, in order to reduce the resistive loss of the active circuit while maintaining a stable behaviour and a wideband matching. The realized antenna of $k a=0.31$ is matched in a $50 \%$ bandwidth $([0.87-1.45 G H z])$, has a maximal total efficiency of $25 \%$ and a peak realized gain of $-3.6 \mathrm{dBi}$ at $0.93 \mathrm{GHz}$ which is more than $10 \mathrm{~dB}$ higher than the unloaded antenna.

\section{Antenna with Ideal Negative Capacitance}

The geometry of the unloaded antenna is shown in Fig.1. The antenna is a bent monopole integrated on a PCB and mounted on a Rogers RO5088 substrate $\left(\epsilon_{r}=2.2\right.$, $\tan \delta=$ $0.0009)$. The overall dimensions of the structure is $(90 \mathrm{~mm} \times$ $35 \mathrm{~mm}$ ) while the radiating part is confined to $(10 \mathrm{~mm} \times$ $23 \mathrm{~mm})$. The input reflection coefficient and impedance behaviour of the antenna are shown in Fig.2. The antenna resonates at $2.2 \mathrm{GHz}$, while having a significant capacitive reactance below this frequency.

In order to miniaturize the antenna, its capacitive reactance should be compensated. The surface current distribution of the antenna at $1 \mathrm{GHz}$ is given in Fig.3a. Following the distributed matching methodology presented in [33], an internal port (port 2) is defined at $3.8 \mathrm{~mm}$ from the feeding point (Fig.3b). At this position, the antenna exhibits a relatively high surface current distribution. Therefore a load placed at this position can efficiently control the current of the antenna in order to match it in a wideband. Using this method, the optimal capacitive value needed to miniaturize the antenna and match it in a lower frequency band is found to be $C=-0.87 p F$. With this negative capacitance connected at the second port, the antenna is matched in a wide bandwidth $[1.06-1.44 G \mathrm{~Hz}]$.

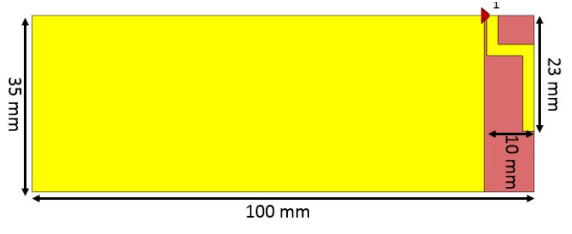

Fig. 1. Geometry of the unloaded antenna

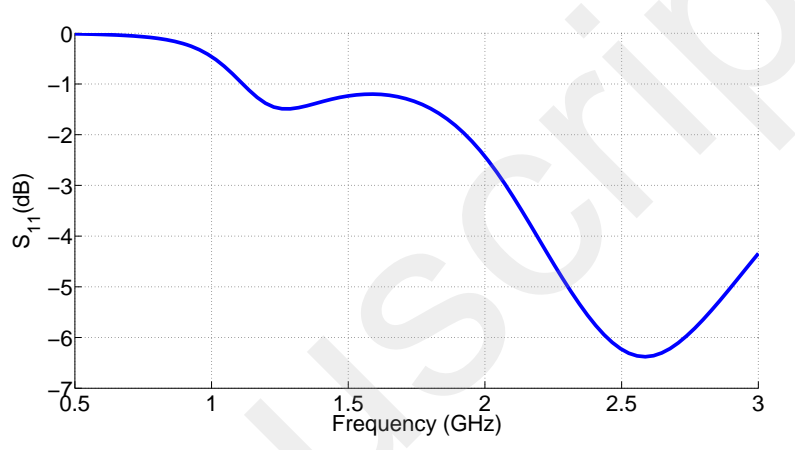

(a)

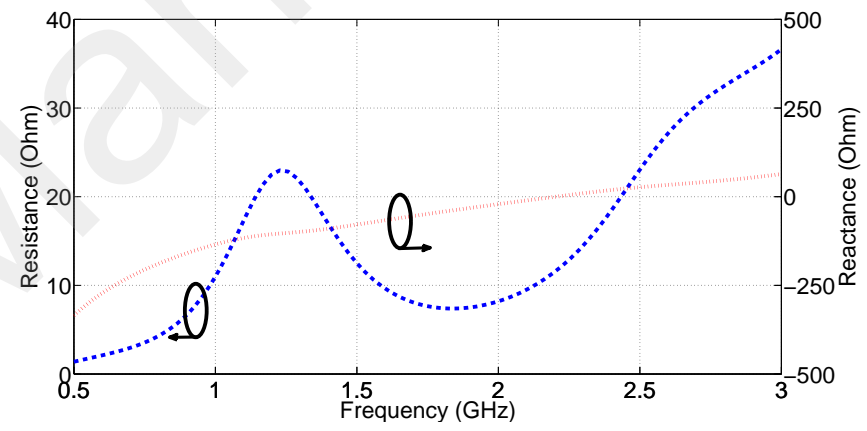

(b)

Fig. 2. Simulated antenna parameters (a) Input reflection coefficient, (b) input impedance.

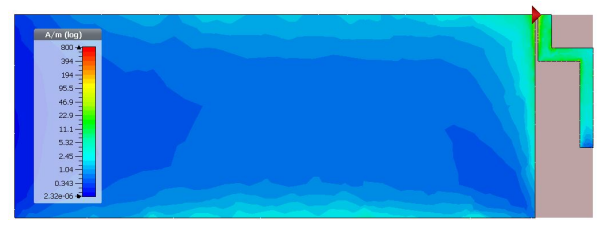

(a)

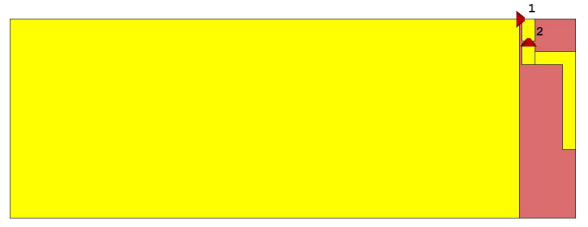

(b)

Fig. 3. (a) Current distribution of the antenna at $1 G H z$, (b) geometry of the two port antenna

This represents $30 \%$ of the central frequency. The antenna's resonance frequency shifts to $1 \mathrm{GHz}$ at which the antenna has an electric size $k a=0.31(k a=1.2$ considering the dimensions of the ground plane). Hence the negative capacitive 


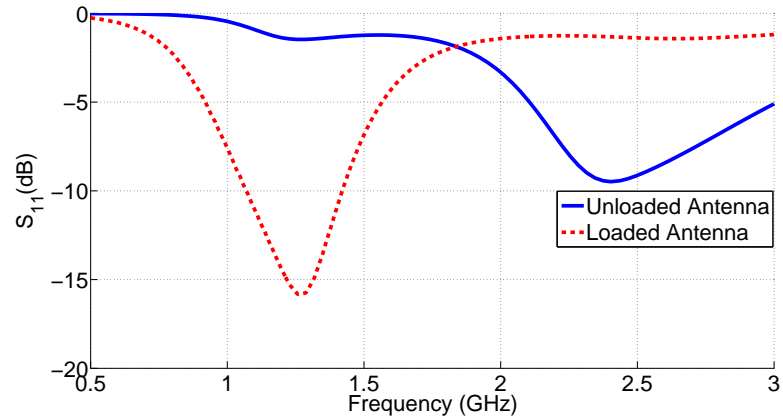

Fig. 4. Input reflection coefficient of the loaded and unloaded antennas.

load miniaturizes the antenna by a factor of 2.2. A comparison between the input reflection coefficients of the loaded and unloaded antennas is shown in Fig.4.

In the next section we present the integration of the realistic non-Foster circuit inside the antenna.

\section{NIC CIRCUIT TOPOLOGY}

After determining the ideal non-Foster load value that is capable to miniaturize and match the antenna in a wideband, in this section we present the NIC circuit design. To obtain a negative capacitance, a Linvill floating type NIC circuit is considered. The schematic of the circuit is shown in Fig.5. Two BJT transistors connected through a feedback path with a resistance $R_{L}$. The transistors used are BFR93A. Rather than including a capacitance in the feedback path, the parasitic capacitance of the transistors is used to generated the desired negative capacitance. Fig. 5b shows the capacitive behaviour of the circuit. It is evident that the circuit generates a negative capacitance over the whole band. In the band of interest, the value of the capaciatance is almost equal to $-0.87 p F$ which corresponds to the optimized value required to match the antenna in a wideband. The initial parameters of the circuit components is given in Table. I.

TABLE I

INITIAL CIRCUIT PARAMETERS.

\begin{tabular}{|c|c|c|c|}
\hline $\mathrm{C} 1$ & $100 \mathrm{pF}$ & $\mathrm{Ls}$ & $470 \mathrm{nH}$ \\
\hline $\mathrm{Cs}$ & $33 \mathrm{nF}$ & $\mathrm{Rs}$ & $100 \mathrm{Ohm}$ \\
\hline $\mathrm{Cb}$ & $100 \mathrm{pF}$ & $\mathrm{Rb}$ & $100 \mathrm{Ohm}$ \\
\hline
\end{tabular}

\section{Antenna With InTEgRATEd NIC CiRCUit}

After choosing the topology of the NIC circuit, the next step is to integrate the circuit inside the antenna. However in order to obtain accurate results, the EM model of the NIC circuit is taken into account in the full wave simulation of the antenna. This EM model will provide a better understanding on the electromagnetic interaction between the antenna and the embedded circuit.In this section, we study the effect of the circuit position on the antenna's matching and efficiency. Moreover, a parametric study of the key circuit components is carried out to optimize the overall system performance. Two cases are taken into account, in which we show that the

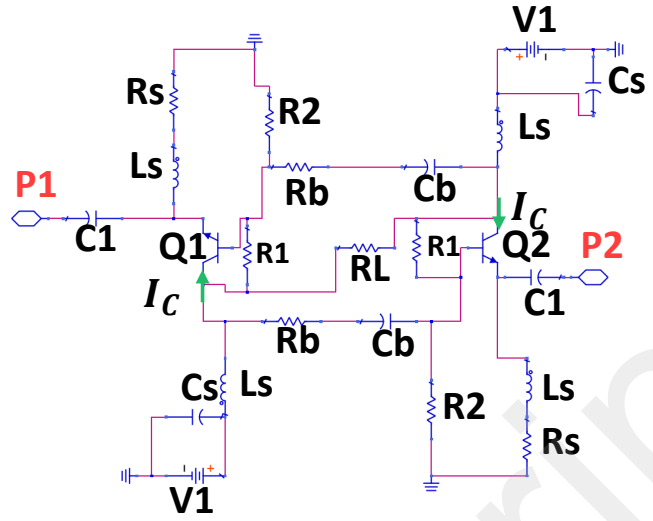

(a)

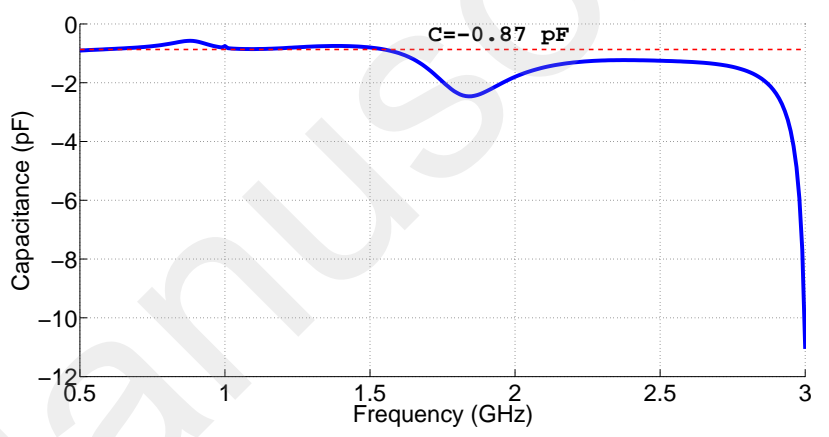

(b)

Fig. 5. The proposed NIC circuit (a) Schematic, (b) de-embedded capacitance.

topology of the embedded NIC can have an impact on the impedance bandwidth and efficiency of the antenna.

\section{A. case 1}

The initial geometry of the anetnna with the NIC circuit is given in Fig. 6. Due to the dimensions of the NIC circuit, the first arm of the monopole is increased by $5 \mathrm{~mm}$ in order to properly fit the NIC inside the given dimensions (Fig. 6a). This increase will just result in shifting the resonance frequency of the antenna. A $0.5 \mathrm{~mm}$ gap is cut in the antenna. At each end of the gap, a via is connected to a terminal of the NIC which is located on the bottom side of the antenna (Fig. 6b). To predict the behaviour of the antenna in the presence of NIC, each component in the circuit is defined as a discrete port, which yields to an overall network of 27 ports. After running the full wave simulation in CST [37],each port in the network is connected to its corresponding circuit element in post processing. The association of the ports to the components is given in Fig. 6.C, B,E corresponds respectively to the collector, base and emitter of the BJT. The input reflection coefficient and the total efficiency of the antenna is given in Fig. 7a. With $I_{c}=2.95 \mathrm{~mA}$ the antenna is matched in a wide band $[1-2.04 G H z](66 \%)$ which is larger than the one with ideal capacitance. However, the overall system efficiency is very low in the whole band $\left(\eta_{\max }=2.7 \%\right)$. In this situation, the EM model of the antenna in the presence of the circuit becomes useful to investigate the main reason of this low efficiency. Fig. $7 b$ shows the surface current distribution of the 


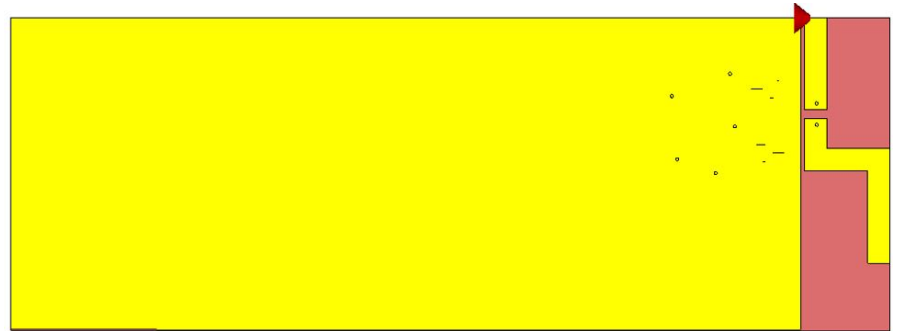

(a)

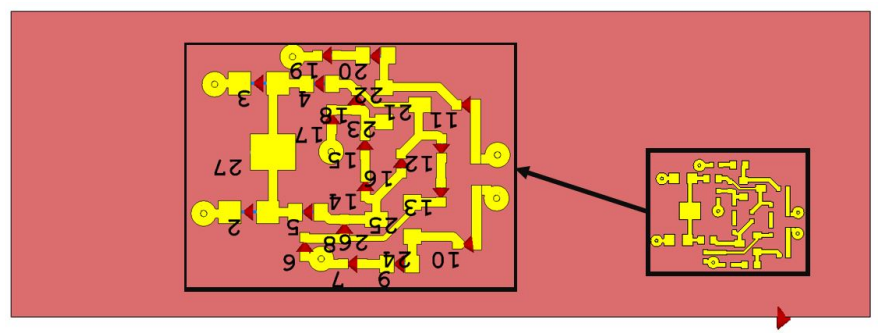

(b)

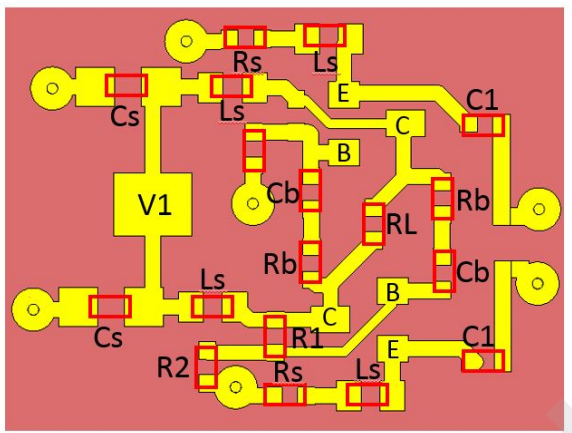

(c)

Fig. 6. Geometry of the antenna with internal NIC(a) Top view, (b) bottom view, (c) EM NIC model with associated elements

embedded NIC at $1 \mathrm{GHz}$. It is obvious that the current inside the NIC is very low. However, an active circuit embedded inside the antenna will become a part of this radiating element, hence a current coming from the antenna and passing inside the NIC is supposed to radiate a certain amount of power. However, a ground plane is placed directly above the NIC which is preventing part of the power to be radiating and therefore resulting in a very low efficiency.

To eliminate this inconvenience and allow the current passing through the NIC to radiate, a gap of size $13 \mathrm{~mm} \times 7 \mathrm{~mm}$ is etched in the ground plane of the antenna, exactly above the circuit. Fig. 8a shows the geometry of the antenna with the font. The substrate is made transparent to visualize the NIC circuit on the bottom side. With this configuration the antenna is matched in a wide bandwidth $[1.05-1.68 G H z](46 \%)$ for $I_{c}=4.7 \mathrm{~mA}$, in addition to an increase in the overall efficiency in the operating bandwidth $\left(\eta_{\max }=9 \%\right)$ at $1.2 \mathrm{GHz}$ which represents an enhancement by a factor of 3 with respect to the initial configuration.

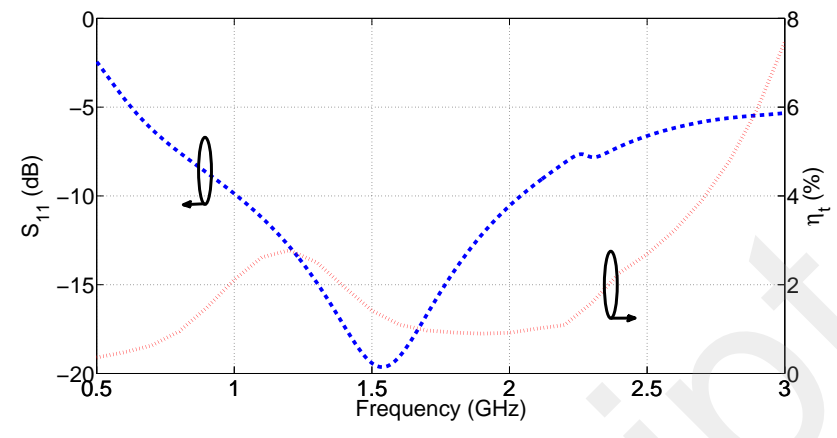

(a)

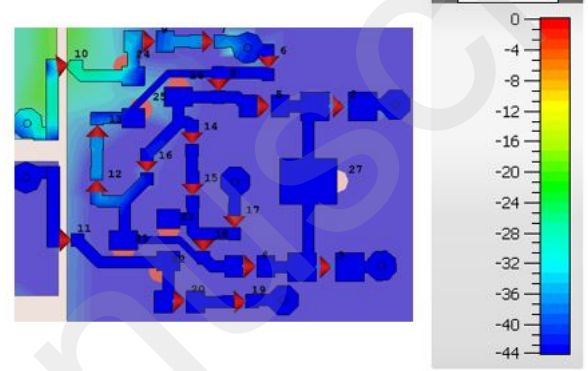

(b)

Fig. 7. Performance of the proposed antenna with active circuit. (a) Input reflection coefficient and efficiency, (b) Surface current distribution of the embedded NIC at $1 \mathrm{GHz}$.

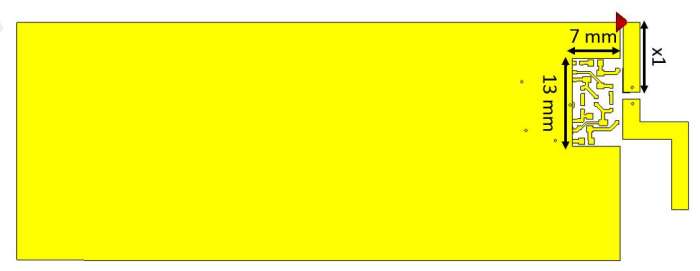

(a)

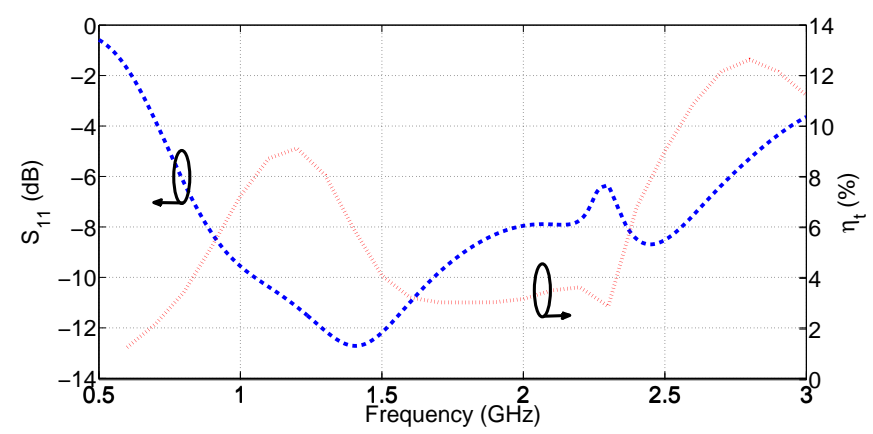

(b)

Fig. 8. Antenna with slot in the ground plane. (a)Geometry of the antenna, (b) input reflection coefficient and efficiency

Fig.9 shows the surface current distribution of the NIC after this modification. After introducing a gap in the ground plane, the surface current distribution in the NIC is significantly increased compared to the results of Fig.7b. This indicates that the current passing from the antenna through the NIC is now capable of radiating the power which was translated in enhancement in the total efficiency. Although the efficiency 

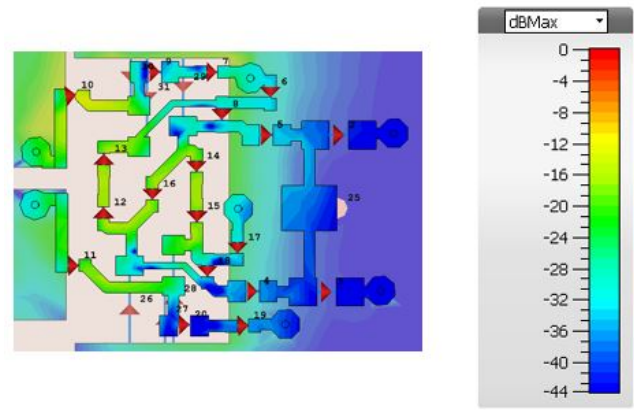

Fig. 9. surface current distribution of the NIC after adding a gap in the ground plane.

TABLE II

PARAMETRIC VALUES OF $R_{b}, L_{s}$, AND $I_{c}$ FOR DIFFERENT STATES

\begin{tabular}{|c|c|c|c|c|c|}
\hline state & $R_{b}(\mathrm{Ohm})$ & $L_{s}(\mathrm{nH})$ & $I_{c}(\mathrm{~mA})$ & $B W(\%)$ & $\eta_{t}(\%)$ \\
\hline 1 & 100 & 470 & 4.7 & 46 & 9 \\
\hline 2 & 60 & 470 & 5.7 & 52 & 10.5 \\
\hline 3 & 20 & 470 & 4.1 & 51.2 & 12 \\
\hline 4 & 20 & 270 & 2.95 & 45 & 13.5 \\
\hline 5 & 20 & 220 & 2.5 & 43.7 & 17 \\
\hline
\end{tabular}

have been enhanced, the achieved value is still relatively low and it is important to investigate the possibility to enhance more this parameter while maintaining a wideband impedance behaviour. Therefore, changing the components inside the circuit might be an interesting option to help reduce the losses introduced by the circuit. Fig.10, shows the current passing from the antenna through the NIC. Ideally the current (red arrow) passing from the antenna to port 1 of the NIC should completely pass from the emitter through the base. However, the choke inductance $L_{s}$ does not provide an ideal isolation and hence part of the current will be lost in the chock inductance as marked by the black arrows. Therefore, a parametric study should be carried on $L_{s}$ to find the best realistic component value that provide the best isolation. This is typically the inductance whose self resonance lies in the frequency band of interest. Another parameter to take into account in our study is the base resistance $R_{b}$, part of the current passing through $R_{b}$ (green arrows) will pass through $R_{L}$. The principle role of $R_{L}$ is to reduce the resistive loss of the circuit, the negative value of $R_{L}$ will be added to the positive resistance of the circuit and will compensate it. However, when the value of $R_{b}$ increases, the voltage across $R_{L}$ causing in reducing its effect in compensating the resistive losses. Therefore, it is also important to carry out a parametric study on $R_{b}$ in order to identify the best value that can help in enhancing the overall performance. Based on this interpretation, the effect of $R_{b}$ and $L_{s}$ in the band of interest between $0.8 \mathrm{GHz}$ and $1.6 \mathrm{GHz}$ was studied by varying their values. Each parametric change of these components is represented by a state, whose values are given in Table.II. It should be noted that changing the values of the components in the NIC might give rise to oscillations causing instabilities. The stability of the active system will be verified in section $\mathrm{V}$ for the different states.

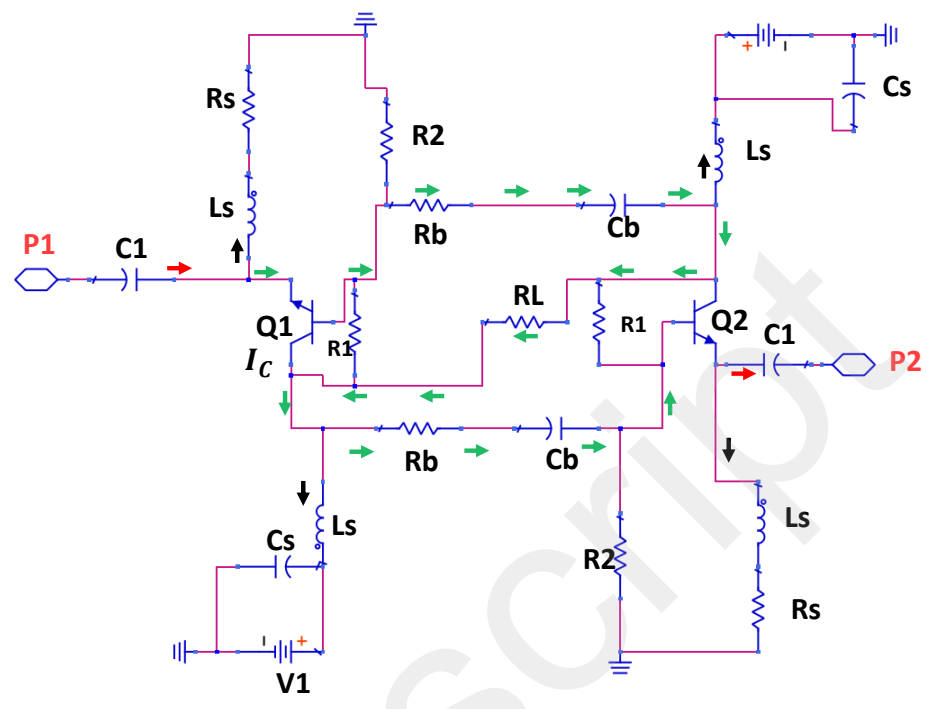

Fig. 10. Current path of the from antenna ports through the NIC:

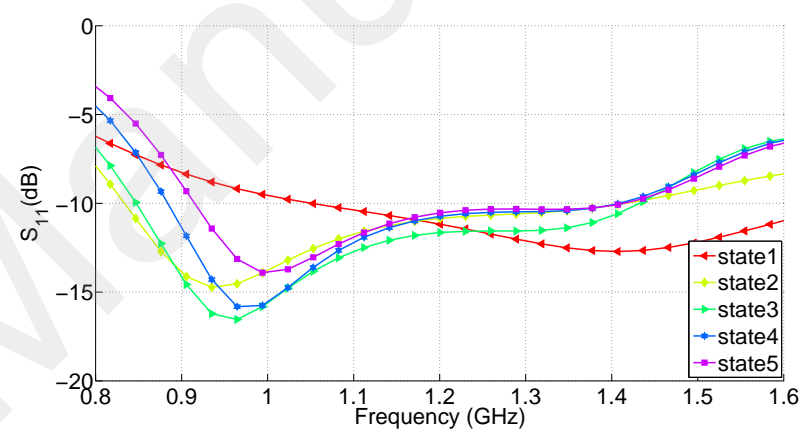

(a)

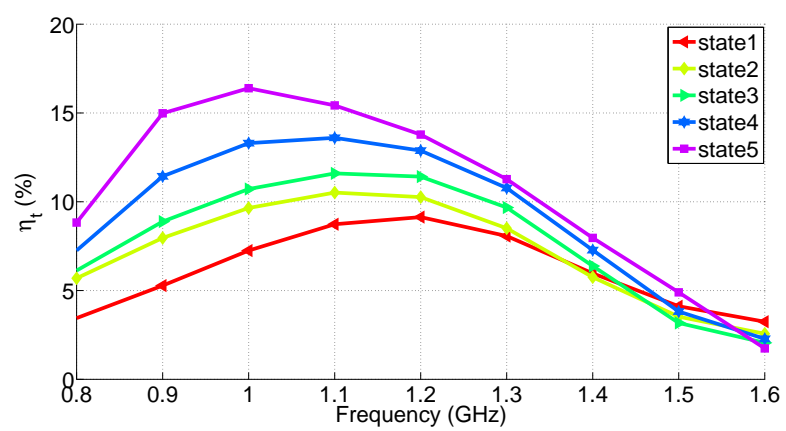

(b)

Fig. 11. Variation of the antenna performance as a function of $R_{b}$ and $L_{s}$. (a) Input reflection coefficient, (b) total efficiency

Fig.11 shows the variation of the antenna performance as a function of $R_{b}$ and $L_{s}$. In the first three states, the value of $L_{s}$ was fixed to $470 \mathrm{nH}$ while the value of $R_{b}$ was decreased from $100 \mathrm{Ohm}$ to $20 \mathrm{Ohm}$. At each state, the DC feed $V 1$ is also varied to ensure the correct excitation of the transistors so that the NIC circuit functions properly. We notice that as the value of the base resistance $R_{b}$ decreases the total efficiency of the circuit increases up to $12 \%$ with a corresponding relative bandwidth of $51.2 \%$. Moreover, while fixing the value of $R_{b}$ to $20 \mathrm{Ohm}$ and decreasing the value 


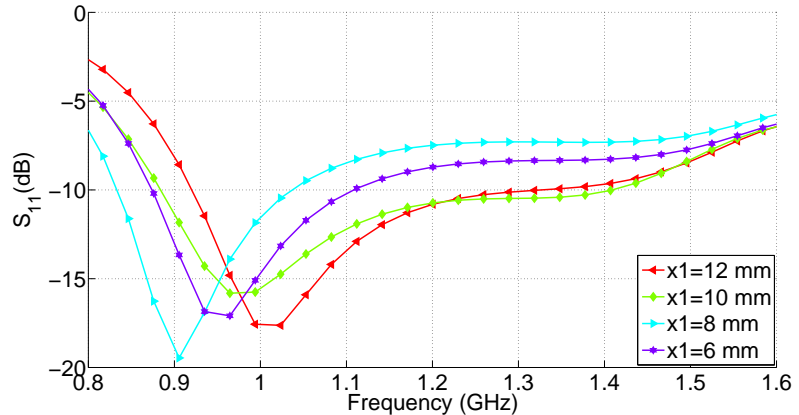

(a)

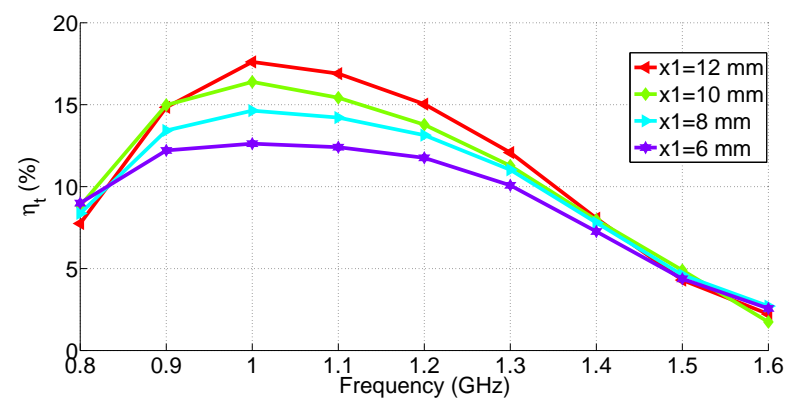

(b)

Fig. 12. Variation of the antenna performance as a function of the NIC position. (a) Input reflection coefficient, (b) total efficiency

of $L_{s}$ from $470 n H$ to $220 n H$, the total efficiency increases up to $17 \%$ with a corresponding relative bandwidth of $43.7 \%$. The enhanced performance of of state 5 compared to state 1 verifies the previous interpretations. Reducing the value of $R_{b}$ from $1000 \mathrm{hm}$ down to $200 \mathrm{hm}$ have increased the ability of the load resistance $R_{L}$ in compensating the resistance of the NIC. Besides, decreasing the value of the inductance from $470 n \mathrm{H}$ to $220 \mathrm{nH}$ have increased the effect of the isolation in the choke inductance. This is due to the fact that the self resonance of the $470 \mathrm{nH}$ inductance is $700 \mathrm{MHz}$ which is lower than that of $220 \mathrm{nH}(1200 \mathrm{MHz})$ [38].Therefore, by performing a parametric study on the values of $R_{b}$ and $L_{s}$ it was possible to increase the total efficiency of the active antenna from $9 \%$ up to $17 \%$ while maintaining a wideband impedance matching. As the values of $R_{b}$ and $L_{s}$ decrease, the resistive effect of the NIC circuit decreases and hence the total efficiency increases. It should be noted that at high frequencies, the parasitic components of the inductance starts to play an improtant role in the overall circuit behaviour, therefore it is improtant to correctly model the inductors in order to accurately predict the overall system performance. In the simulations, the $s 2 p$ touchstone files of the Murata LQW18AN library are used to model $L_{s}$ [38]. This model is also important to take into account the self resonance of the these inductors.

On the other hand, it is also important to study the effect of the circuit position on the overall system performance. Fig. 12 shows the variation of the antenna performance as a function of the circuit position $(x 1)$ with respect to the feeding point with the optimized components values $R_{b}=200 \mathrm{hm}$ and $L_{s}=$
$220 n H$.

As expected the overall efficiency of the antenna drops as the circuit approaches the feeding point. Therefore, as the NIC circuit approaches the feed point, its resistive effect becomes more important, resulting in a decrease in the efficiency. For a distance of $x 1=10 \mathrm{~mm}$ the antenna exhibits the best trade off between bandwidth (43.7\%) and efficiency (17\%).

\section{B. Case 2}

In this section we study the effect of changing the topology of the NIC circuit on the overall system performance. In this case the terminals of the NIC circuit are separated by $8 \mathrm{~mm}$ rather than $2 \mathrm{~mm}$ in the previous section (Fig.13b). In order to integrate the active circuit, the gap of the internal port is increased to $7.5 \mathrm{~mm}$. To compensate the introduced gap, the terminal section of the antenna is enlarged by $7 \mathrm{~mm}$ Fig. 13a. Following the same procedure presented in the previous example, it was found that the antenna exhibits the best performance for $R_{b}=200 h m, L_{s}=220 \mathrm{nH}$, and with a separation distance $x 1=8 \mathrm{~mm}$. This is the maximal separation distance in this case, since the increased gap approaches the terminal of the NIC towards the feed point. With this configuration, the antenna is matched in a wideband $[0.87-1.45 G \mathrm{~Hz}]$ which corresponds to $50 \%$ relative bandwidth. Moreover, a maximal efficiency of $24 \%$ is achieved at $1.2 \mathrm{GHz}$ while the efficiency remains higher than $15 \%$ in most of the band (Fig. 14). Compared to the previous case, the modified NIC topology shows a better performance for both bandwidth and efficiency. So, even a slight change in the NIC topology in terms of the terminal positions and configuration results in a change in performance. Hence, in an actively matched antenna, it is important to take the NIC model in the full wave simulation, in order to determine the best position, topology and component values that can improve the overall performance.

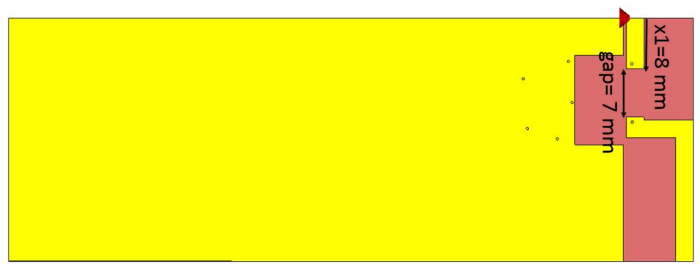

(a)

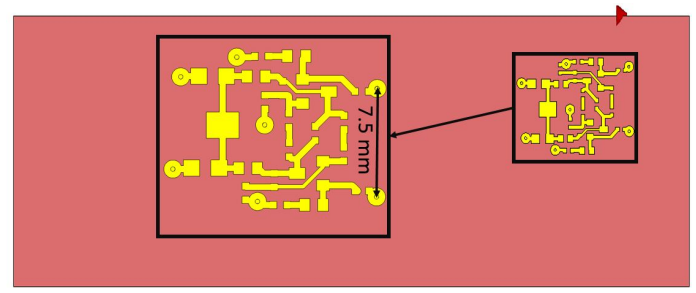

(b)

Fig. 13. Geometry of the antenna with modified NIC topology.

The reason behind this enhancement can be interpreted by analyzing the current distribution in the NIC and the antenna in both cases (Fig.15). The transmission lines of the NIC (case 1) marked in red exhibit a high surface current distribution. These 


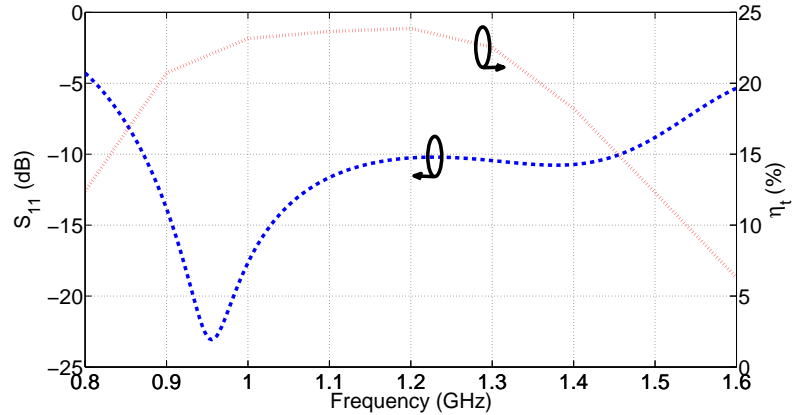

Fig. 14. Input reflection coefficient and total efficiency of the antenna with modified NIC topology.

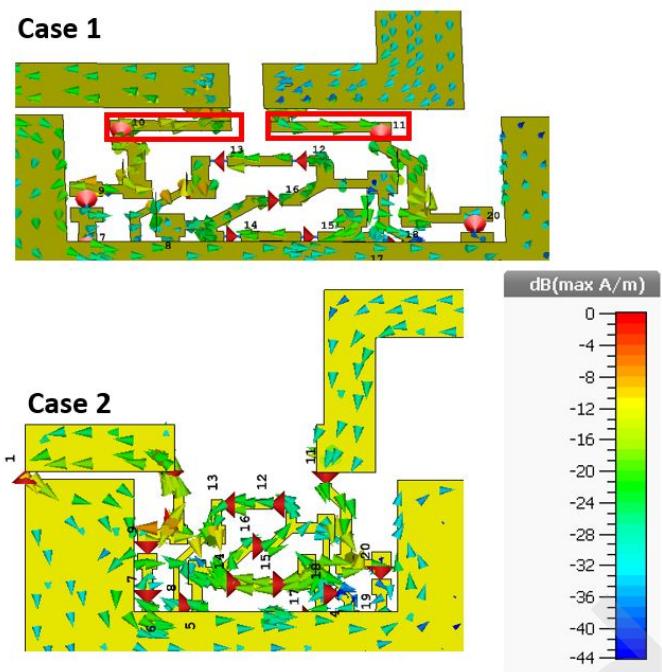

Fig. 15. Surface current distribution of the NIC and the antenna for the different topologies

lines are located directly under the antenna, while having an out of phase current distributions with respect to the antenna. This will result in canceling part of the radiated power leading to a decrease in the efficiency. This explains the enhancement in the efficiency after removing these transmission lines by increasing the gap between the NIC ports. With this new topology the out of phase currents are eliminated (Fig.15)

\section{STABILITy ANALYSIS}

The positive feedback which is necessary to achieve a nonFoster impedance in the NIC imposes a naturally unstable behaviour of this circuit. Therefore, when dealing with an actively macthed antenna using non-Foster impedance, it becomes necessary to study the stability of the whole system to insure a reliable performance [25], [34], [35]. In [36], Middlebrook has introduced a method to measure the loopgain $T$ of a positive feedback system by injecting a small signal voltage and / or current at a test point in the feedback loop and measuring the gain around this loop. This gain can then be used in a Nyquist test to study the existence of Right Hand Plane (RHP) zeros and poles of the denominator $D$ of the system's transfer equation $(D=1+T)$. In our case, the test point is defined in the feedback passing by the base resistance $R_{b}$. At this test point, two tests involving current are voltage injections are considered to calculate the $T$ given by the following equation [36].

$$
T=\frac{T_{v} T_{i}-1}{2+T_{v}+T_{i}}
$$

where $T_{v}$ is the voltage loop-gain and $T_{i}$ is the current loop gain. Fig.16a,b shows the Nyquist plot of $\mathrm{L}$ for the positive frequencies up to $10 \mathrm{GHz}$ which is the maximal frequency for which the models of the circuit components are still valid. Both figures illustrate the variation of $T$ as for different values of $L_{s}$ and $R_{b}$ corresponding to the five parametric states which were considered in Table. II. Fig.16a, shows the response of the antenna with the circuit topology of case 1 while Fig.16b shows that with circuit topology of case 2 . In both cases and for all the studies states, the Nyquist contour does not exhibit encirclement around the point $s=-1+j 0$ and converges to the origin as the frequency approaches $10 G H z$. By analyzing these contours, it can be deduced that $R_{b}$ is the parameter that can potentially affect the stability of the system. for values of $R_{b}$ that are relatively high $\left(R_{b}=1000 \mathrm{hm}\right.$ for state 1 and $600 \mathrm{hm}$ for state 2) the Nyquist contours are closer to the origin for all the positive frequencies in the bandwidth, however, when $R_{b}$ decreases down to $20 \mathrm{Ohm}$ the domain occupied by the contours becomes larger. On the other hand, changing the values of $L_{s}$ does not have a huge impact on the stability. Due to the fact that there is no Clock-wise encirclement around $s=-1+j 0$ we can assume that this system is supposed to be stable for all the studied parametric states, the stability of the final design will be verified in the next section by analyzing its spectrum in a wide frequency range.

The effect of decreasing $R_{b}$ below $200 h m$ on the stability of the system is shown in Fig.16c. In this case $R_{b}$ was set to zero, and $L_{s}=220 \mathrm{nH}$. The Nyquist contour was plotted for both cases. By analyzing the plot, it can be seen that for both cases, there exists an inflection point at which the contour changes its direction and circulates in a counter clock-wise direction to the left of the point $s=-1+j 0$ this behvaiour indicates the possibility of existence of poles in $D$ which gives rise to oscillations and instabilities. This observation, will also be verified in the measurements of the spectrum of the antenna when $R_{b}=0 O h m$. 


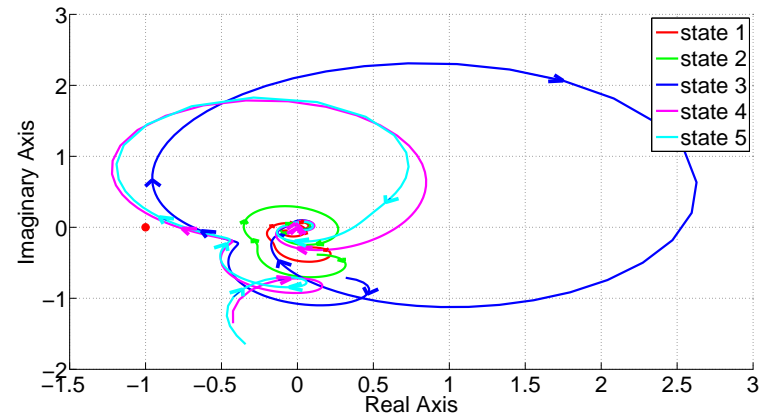

(a)

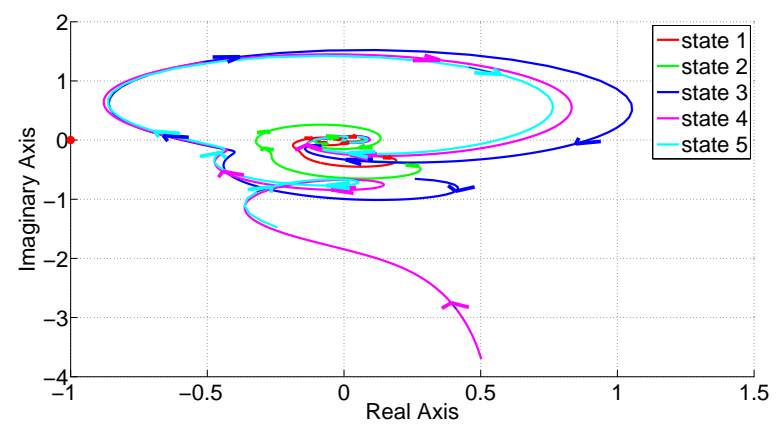

(b)

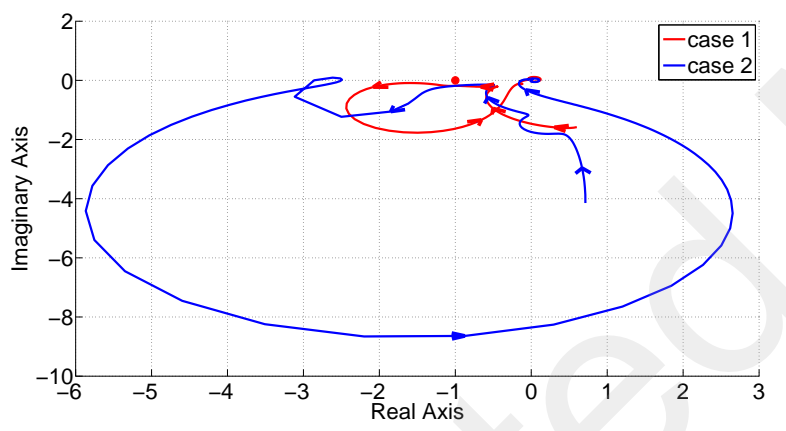

(c)

Fig. 16. Nyquist plot of the loop-gain for positive frequencies up to $10 \mathrm{GHz}$ (a) Case 1, (b) case 2, (c) base resistance equals zero for both cases.

\section{EXPERIMENTAL VALIDATION}

Two prototypes of the previous cases have been fabricated. The prototype of case 1 is shown in Fig. 17 with the antenna printed on the top side and the NIC circuit on the bottom connected through vias. As stated in section II, the antenna is printed on a Rogers RO5088 substrate $\left(\epsilon_{r}=2.2\right.$, $\tan \delta=$ $0.0009)$. The measured input reflection coefficient of both cases is shown in Fig. 18. For case 1, the measured active antenna is driven by $I_{c}=2.95 \mathrm{~mA}$ and is matched in a wideband $[1.02-1.63 \mathrm{GHz}]$ which represents $45.6 \%$ relative bandwidth. This result agrees with the simulated antenna driven by the same DC voltage and having a relative bandwidth of $43.7 \%$. However, the measured antenna exhibits a shift in the resonance frequency with respect to the simulation. Moreover, the measured results of the case 2 are also in good agreement with the simulation. The measured antenna driven by $I_{c}=2.5 \mathrm{~mA}$ is wideband and matched in $58.7 \%$ relative bandwidth $([0.9-1.66 \mathrm{GHz}])$. It should be noted that using the

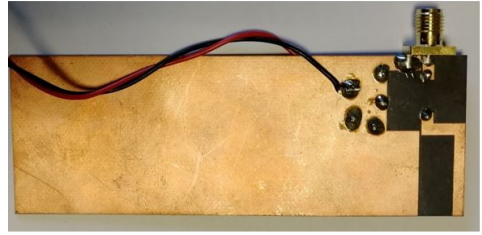

(a)

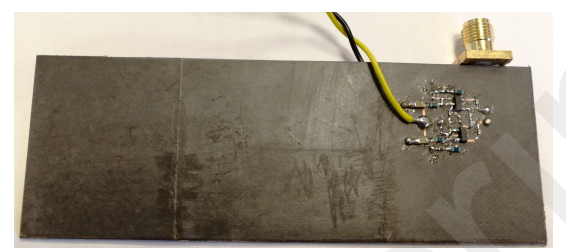

(b)

Fig. 17. Geometry of the antenna with modified NIC topology.

NIC circuit, the antenna achieves a wider bandwidth compared to the ideal capacitance case presented section II. This is due to the existing resistive loss in the circuit that causes this increase in the bandwidth. On the other hand, the measured efficiency of both cases is presented in Fig. 19. As predicted in simulation, the antenna of case 1 attains a maximal efficiency of $18 \%$ and a $23 \%$ for case 2 .

Fig. 20a shows the comparison between the realized gain of the passive antennas and the active antennas. In its bandwidth of operation, the antenna of case 1 achieves a peak gain of $-4.6 \mathrm{~dB}$ at $1.05 \mathrm{GHz}$ while that of case 2 achieves a peak gain of $-3.5 \mathrm{~dB}$ at $0.93 \mathrm{GHz}$. In both cases, the realized gain is significantly better than the passive antenna which has a gain less than $-10 d B$ at the previously mentioned frequencies. Furthermore, the gain of the active antennas is also higher than the actively matched antenna designed in [15] and having the same size, which proves the advantage of internal active matching rather than input matching in this frequency band. On the other hand, the quality factor of the measured passive and active antennas is shown in Fig.20b. A dramatic decrease is noticed in the $\mathrm{Q}$ factor of the antenna when the nonFoster circuit is connected. As expected, the antenna of case 2 presents a slightly lower $\mathrm{Q}$ with respect to the antenna of case 1 especially in the lower part of the band. This decay in the Q factor between the passive and active antennas, proves that the negative capacitance effectively compensated the reactance of the electrically small monopole making it more suitable to be matched in a wideband.

To verify the stability prediction which was studied in section $\mathrm{V}$, the prototype of case 2 was connected to a spectrum analyzer to insure that no oscillations are produced in the active system. Fig.21, shows the spectral behavior of the prototype for two cases: $R_{b}=200 \mathrm{hm}$, and $R_{b}=00 \mathrm{hm}$ while maintaining a similar value of $L_{s}=220 n H$. The parameters of the first case corresponds to that of state 5 which was proven to offer the best bandwidth-efficiency trade-off. The spectrum is studied from 0 up to $20 \mathrm{GHz}$. For $R_{b}=200 \mathrm{hm}$, no oscillations are observed in the spectrum, which verifies the stability analysis of the Nyquist plots. The instability due to low $R_{b}$ values is also verified by the oscillation that are present 

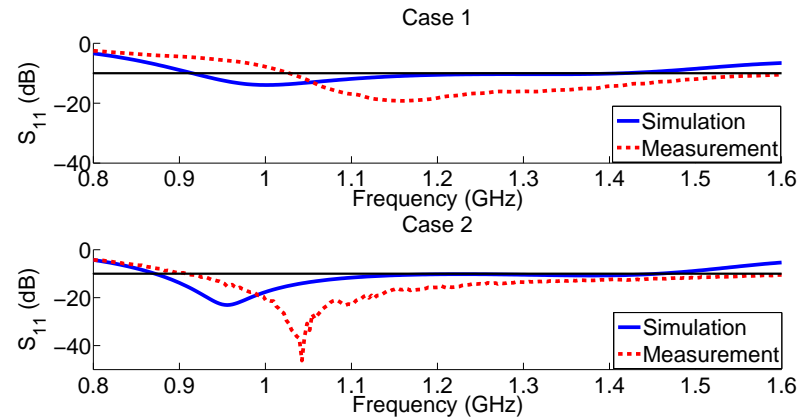

Fig. 18. Input reflection coefficients of the measured active antennas
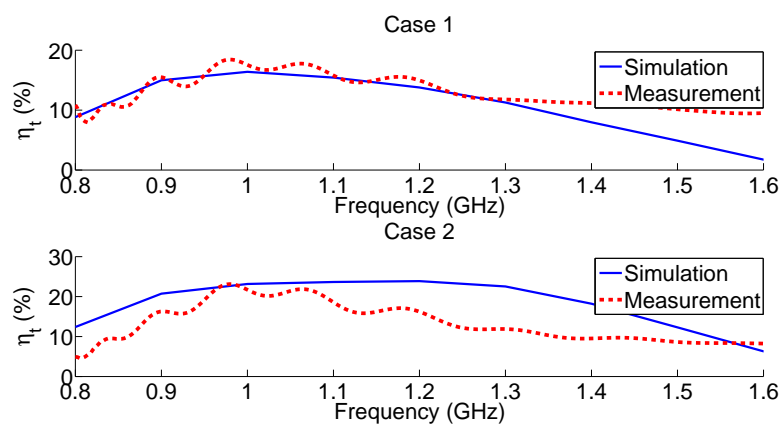

Fig. 19. Total efficiency of the active antennas.

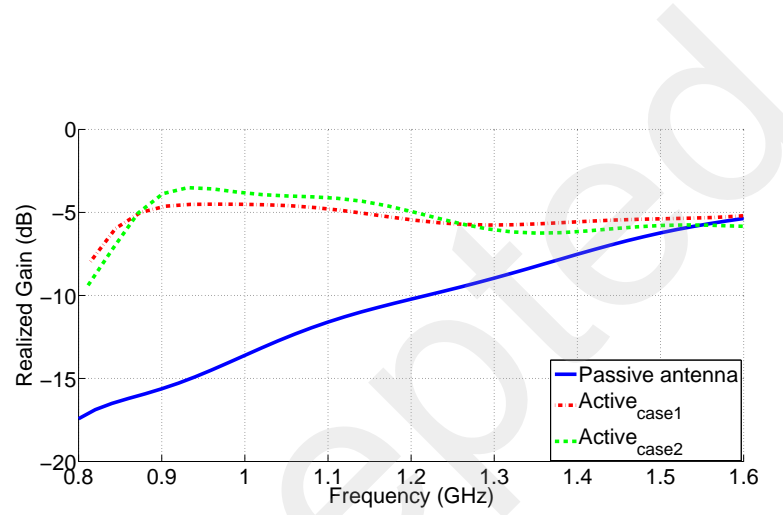

(a)

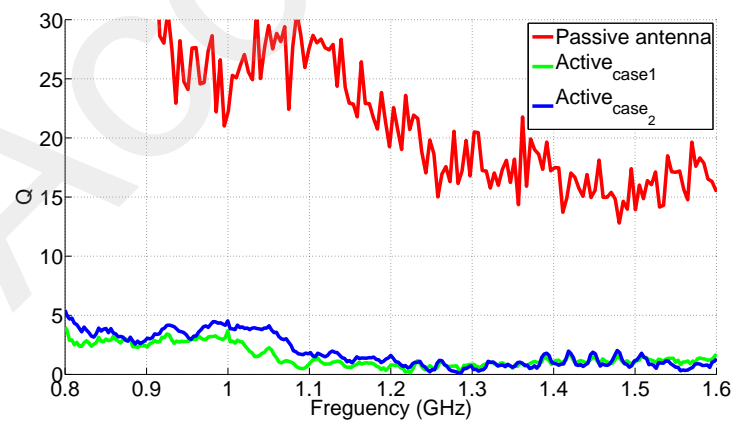

(b)

Fig. 20. Comparison between the internally loaded antennas and the original antenna.(a) Realized gain, (b) Q factor.

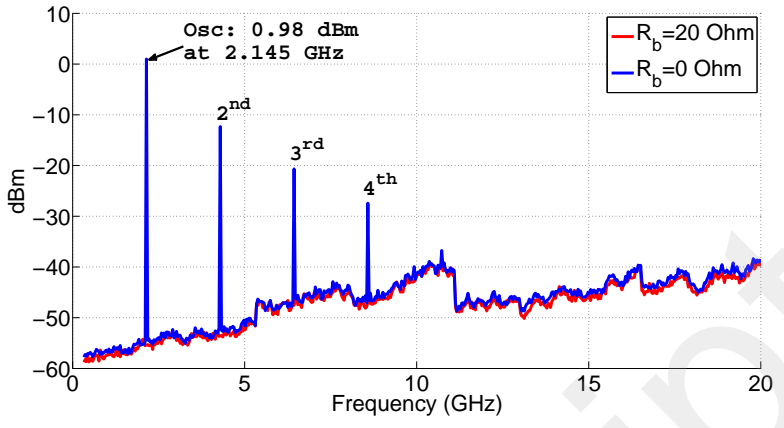

Fig. 21. Spectral analysis of the antenna of case 2 for stable and unstable behaviours.

in the spectrum. For this value of $R_{b}$, the circuit oscillates at $2.14 G H$ which is the fundamental frequency of oscillation and the corresponding harmonics are also visible. Therefore, with the given NIC topology, a minimal value of $R_{b}$ is important to insure a stable circuit operation.

\section{CONCLUSION}

This paper presents the design of an active internally matched electrically small antenna. The original passive antenna resonates at $2.2 \mathrm{GHz}$. Using an ideal non-Foster capacitance embedded inside the antenna,it was shown that it is possible to miniaturize the antenna by a factor 2.2 where $k a=0.3$ and match it in a wideband $(30 \%)$. However, the realization of a negative capacitance is associated with NIC circuits which are sensitive and introduce resistive losses. Therefore, we propose modeling the NIC circuit with the antenna in the full wave simulation in order to have more accurate results and optimized performance. Each component of the NIC is defined as a discrete port and finally connected to its corresponding element in the post processing. It was noticed that by introducing a slot exactly above the NIC circuit, the total efficiency was increased by approximately a factor of 3 (from $2.7 \%$ up till $9 \%$ ). Moreover, using the post processing in CST, a parametric study have been carried to identify the key components in the NIC that can affect the total efficiency of the system. It was then shown that for an $R_{b}=20 \mathrm{Ohm}$ and $L_{s}=220 n H$, a maximal efficiency of $17 \%$ was achieved with an impedance bandwidth of $43.7 \%$. Furthermore, the position of the circuit inside the antenna was varied to show its effect on the overall performance. It was shown that as the NIC approaches the feed point, the overall efficiency drops. This is due to the NIC's resistive loss which becomes more important as it gets closer to the antenna's feed. Following the same procedure, another case have been considered in which the distance between NIC's terminals is increased. In this case, the antenna shows a better performance than the previous one with a $24 \%$ efficiency and $50 \%$ bandwidth. Measurement results are in good agreement with the simulations in both cases. The realized gain of the active antenna shows a significant enhancement with respect to the passive antenna.

Therefore, using the EM modeling of the NIC in the presence of the antenna it is possible to predict the overall 
behaviour of the system and to carry out parametric studies in order to optimize the performance. In addition, internal active matching is advantageous compared to input active matching where the resistive effect of the NIC becomes more important.

In theory, it is possible to enhance more the bandwidth of the antenna up to higher than $100 \%$. However, this might require multiple non-Foster loads distributed along its geometry. While in an ideal scenario, this can provide a very efficient and wideband antenna, practically this adds a huge complexity to the system in addition to the limited space available especially for a physically small antenna. Therefore, the initial antenna geometry remains an important point in obtaining a desired behaviour.

\section{ACKNOWLEDGMENT}

This work is supported by the European Union through the European Regional Development Fund (ERDF), and by Ministry of Higher Education and Research, Brittany and Rennes Métropole, through the CPER Project SOPHIE / STIC \& Ondes, and by the Direction Général de l'Armement (DGA).

\section{REFERENCES}

[1] J.G. Linvill, "Transistor negative impedance converters "Proc IRE 41 pages $725-729,1953$.

[2] C. K. Kuo and K. L. Su, "Some new four terminal NIC circuits," IEEE Proc. Circuit Theory, pp. 379-381, Aug. 1969.

[3] L. J. Chu, "Physical limitations of omni-directional antennas," $J$. Appl.Phys., vol. 19, no. 12, pp. 11631175, Dec. 1948.

[4] J.H. Wheeler, "Fundamental Limitations of Small Antennas," Proceedings of IRE, vol. 35, December 1947, pp. 1479-1484.

[5] H. W. Bode, "Network Analysis and Feedback Amplifier Design", New York, NY, USA: Van Nostrand, 1945.

[6] R. M. Fano, "Theoretical limitations on the broadband matching of arbitrary impedances,"J. Franklin Inst., vol. 249, pp. 139154, 1950.

[7] K. A. Obeidat, B. D. Raines and R. G. Rojas, " Application of Characteristic Modes and Non-Foster Multiport Loading to the Design of Broadband Antennas," in IEEE Transactions on Antennas and Propagation, vol. 58, no. 1, pp. 203-207, Jan. 2010.

[8] H. Mirzaei, G. V. Eleftheriades, "Realizing non-Foster reactive elements using negative-group-delay networks", IEEE Transactions on Microwave Theory and Techniques, vol. 61, no. 12, pp. 4322-4332, Dec. 2013.

[9] S. Hrabar, I. Krois, I. Bonic, A. Kiricenko, Negative Capacitor Paves the way to Ultra-broadband Metamaterials, Appl. Phys. Lett., 99, 25, (2011), 25403 25403-4

[10] M. M. Jacob, J. Long and D. F. Sievenpiper,"Non-Foster Loaded Parasitic Array for Broadband Steerable Patterns,"IEEE Transactions on Antennas and Propagation, vol. 62, no. 12, pp. 6081-6090, Dec. 2014.

[11] H. Jaafar, A. Sharaiha and S. Collardey,"Design of a wideband superdirective endfire antenna array using characteristic modes optimization," 2017 XXXIInd General Assembly and Scientific Symposium of the International Union of Radio Science (URSI GASS), Montreal, QC, 2017, pp. $1-4$.

[12] L. Batel, L. Rudant, J. F. Pintos, A. Clemente, C. Delaveaud and K. Mahdjoubi, "High directive compact antenna with non-foster elements," 2015 International Workshop on Antenna Technology (iWAT), Seoul, 2015, pp. 381-384.

[13] J.T. Aberle, R. Loepsinger-Romak, "Antennas with Non-Foster Matching Networks,"in Antennas with Non-Foster Matching Networks ,1, Morgan \& Claypool, 2007.

[14] S. E. Sussman-Fort and R. M. Rudish, "Non-Foster Impedance Matching of Electrically-Small Antennas," in IEEE Transactions on Antennas and Propagation, vol. 57, no. 8, pp. 2230-2241, Aug. 2009.

[15] Abdullah Haskou, Dominique Lemur, Sylvain Collardey, Ala Sharaiha, "Small Wide-Band Printed Inverted-L Antenna with Non-Foster Matching,"IEICE Transactions on Communications vol E101.B, no. 2, pp. 309315 .
[16] D. S. Nagarkoti, Y. Hao, D. P. Steenson, L. Li, E. H. Linfield and K. Z. Rajab, "Design of Broadband Non-Foster Circuits Based on Resonant Tunneling Diodes," in IEEE Antennas and Wireless Propagation Letters, vol. 15, pp. 1398-1401, 2016.

[17] M. M. Jacob and D. F. Sievenpiper, "Design, validation and tradeoffs of non-foster circuits," 2017 IEEE MTT-S International Microwave Symposium (IMS), Honololu, HI, 2017, pp. 344-347.

[18] S. Koulouridis and J. L. Volakis, "Non-foster circuits for small broadband antennas," 2009 IEEE Antennas and Propagation Society International Symposium, Charleston, SC, 2009, pp. 1-4.

[19] A. M. Elfrgani and R. G. Rojas, "Non-Foster circuit embedded within electrically small antenna,"2014 IEEE Antennas and Propagation Society International Symposium (APSURSI), Memphis, TN, 2014, pp. 466-467.

[20] L. Batel, L. Rudant, J. F. Pintos and K. Mahdjoubi,"Sensitivity of negative impedance converter circuit with respect to PCB design effects,"2015 International Workshop on Antenna Technology (iWAT), Seoul, 2015, pp. 221-224.

[21] S. Stefanopoulos and S. Koulouridis, "Realizing non-Foster circuits for antennas," 2013 IEEE Antennas and Propagation Society International Symposium (APSURSI), Orlando, FL, 2013, pp. 1960-1961.

[22] G. Skahill, R. M. Rudish, and J. A. Pierro, "Apparatus and method for broadband matching of electrically small antennas", US Patent 6121940 , Sep., 2000.

[23] F. Albarracn-Vargas, V. Gonzalez-Posadas, F. J. Herraz-Martinez and D. Segovia-Vargas, "Design Method for Actively Matched Antennas With Non-Foster Elements," in IEEE Transactions on Antennas and Propagation, vol. 64, no. 9, pp. 4118-4123, Sept. 2016.

[24] J. Church, J. C. S. Chieh, L. Xu, J. D. Rockway and D. Arceo, "UHF Electrically Small Box Cage Loop Antenna With an Embedded NonFoster Load," in IEEE Antennas and Wireless Propagation Letters, vol. 13, pp. 1329-1332, 201.

[25] H. Mirzaei and G. V. Eleftheriades, "A Resonant Printed Monopole Antenna With an Embedded Non-Foster Matching Network,'IEEE Transactions on Antennas and Propagation, vol. 61, no. 11, pp. 5363-5371, Nov. 2013.

[26] P. Jin and R. W. Ziolkowski, "Broadband, Efficient, Electrically Small Metamaterial-Inspired Antennas Facilitated by Active Near-Field Resonant Parasitic Elements," in IEEE Transactions on Antennas and Propagation, vol. 58, no. 2, pp. 318-327, Feb. 2010.

[27] E. A. Elghannai, B. D. Raines and R. G. Rojas, "Multiport Reactive Loading Matching Technique for Wide Band Antenna Applications Using the Theory of Characteristic Modes," IEEE Transactions on Antennas and Propagation, vol. 63, no. 1, pp. 261-268, Jan. 2015

[28] H. Jaafar, S. Collardey, D. Lemur, A. Haskou and A. Sharaiha, “ Characteristic modes optimisation approach to design a wideband electrically small antenna," 2017 11th European Conference on Antennas and Propagation (EUCAP), Paris, 2017, pp. 2184-2186.

[29] C. Di Nallo, G. Bit-Babik and A. Faraone, "Wideband antenna using non-foster loading elements," 2007 IEEE Antennas and Propagation Society International Symposium, Honolulu, HI, 2007, pp. 4501-4504.

[30] M. C. Tang and R. W. Ziolkowski, "Electrically small metamaterialinspired antennas with active near field resonant parasitic elements: From theory to practice," 2017 11th European Conference on Antennas and Propagation (EUCAP), Paris, 2017, pp. 1559-1563.

[31] F. Albarracin-Vargas and D. Segovia-Vargas, "A printed monopole loaded with a non-Foster matching network in the VHF band; radiation considerations," 2015 9th International Congress on Advanced Electromagnetic Materials in Microwaves and Optics (METAMATERIALS), Oxford, 2015, pp. 7-9.

[32] M. C. Tang, T. Shi and R. W. Ziolkowski, "Electrically Small, Broadside Radiating Huygens Source Antenna Augmented With Internal Non-Foster Elements to Increase Its Bandwidth," in IEEE Antennas and Wireless Propagation Letters, vol. 16, pp. 712-715, 2017.

[33] H. Jaafar, S. Collardey and A. Sharaiha, "Optimized Manipulation of the Network Characteristic Modes for Wideband Small Antenna Matching," in IEEE Transactions on Antennas and Propagation, vol. 65, no. 11, pp. 5757-5767, Nov. 2017

[34] Q. Tang, H. Xin, "Stability Analysis of Non-Foster Circuit Using Normalized Determinant Function", IEEE Tran. Microw. Theory and Techn.,vol. 65,no. 9,pp. 3269-3277, Sep 2017.

[35] S. D. Stearns, "Circuit stability theory for non-Foster circuits," 2013 IEEE MTT-S International Microwave Symposium Digest (MTT), Seattle, WA, 2013, pp. 1-3.

[36] R.D. Middelbrook, “ Measurement of loop gain feedback systems”, Int. J. Electron., vol. 38, no. 4, pp. 485-512, 1975.

[37] CST STUDIO SUITE, CST AG, Germany, www.cst.com. 
[38] Online.Available: eu/tool/sparameter/inductor https://www.murata.com/en-

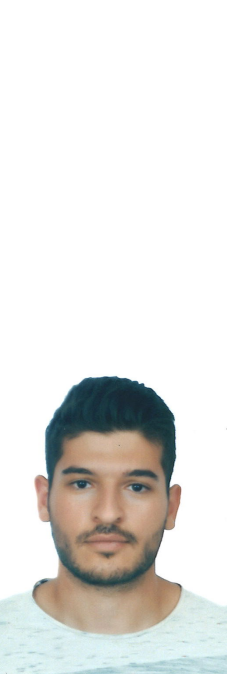

Hussein Jaafar Recieved the B.E. degree in computer and communications engineering and the M.Sc. in microwave engineering from the Lebanese University, Beirut, Lebanon, in 2015. In 2018 he recieved the $\mathrm{PhD}$ degree from the University of Rennes 1 where he studied the design of wideband and superdirective antennas using the characteristic modes theory. Recently, he joined The French Alternative Energies and Atomic Energy Commission (CEA) where he is currently working on the design of innovative miniature antenna for various applications. His research interests include electrically small antennas, distributed matching techniques, the characteristic modes theory, RF circuits design, and RFID.

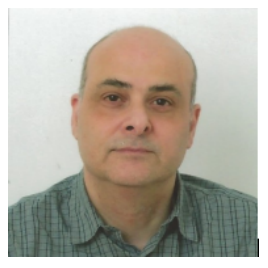

Ala Sharaiha received the Ph.D. and Habilitation Diriger la Recherche (HDR) degrees in telecommunication from the University of Rennes 1, France, in 1990 and 2001 respectively. Currently, he is a Full Professor at the University of Rennes 1 and the Co-Head of the Antennas and Microwave Devices Department at the IETR research Laboratory (Institute of Electronics and Telecommunications in Rennes). He has graduated/mentored more than 34 $\mathrm{Ph} . \mathrm{D}$. students/postdocs and co-authored with them. $\mathrm{He}$ has authored or co-authored more than 400 journal and conference papers, concerning antenna theory, analysis, design and measurements. He holds 12 patents. His published works have been cited over 1500 times in Google Scholar. His current research interests include small antennas, broadband and UWB antennas, reconfigurable antennas, printed spiral and helical antennas, and antennas for mobile communications.

$\mathrm{He}$ is presently a French delegate member of the European Association on Antennas and Propagation (EuRAAP) and a member of the small antennas working group of EuRAAP. He is a senior member of the IEEE and is a reviewer for the IEEE APS, IEEE AWPL, the IET Letters and the IET Microwave Antennas Propagation. He was the conference Chairman of the 11th International Canadian Conference ANTEM (Antenna Technology and Applied Electro- Magnetics), held at Saint-Malo in France, 2005.

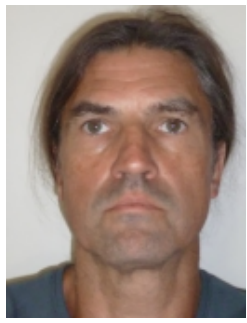

Dominique Lemur received the $\mathrm{Ph} . \mathrm{D}$. degree in electronics from the Universit de Rennes, in 1996. Since 1998, he is associate professor at the Universit de Rennes and is a member of the Institut d'Electronique et de Tlcommunications de Rennes (IETR). His research topics are radio waves propagation and radiolocation in the HF and V/UHF bands. He is particularly involved in ionospheric radio applications for DOA / SSL and improvement in data bit rates with HF SIMO and MIMO modems. He has designed numerous radiofrequency systems, transmitting or active receiving antennas and compacts receiving HF arrays.

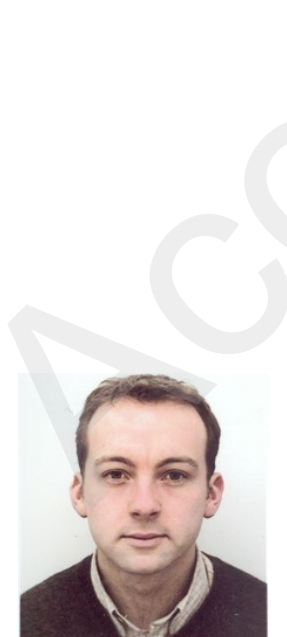

Sylvain Collardey Sylvain Collardey received his $\mathrm{PhD}$ Degree in telecommunication from the University of Rennes 1, France, in 2002. He was graduated in Electronics and Telecommunication Engineering at the University of Rennes 1 in the year 1998 . Currently, he is an associate professor at the University of Rennes 1 and involved as researcher in the Antennas and Microwaves group at the Institute of Electronics Telecommunications of Rennes (IETR). His current research interests include the characterization and development of small antennas, EBG materials and metamaterials, and RFID. He has published more than hundred revue papers and conference communications 